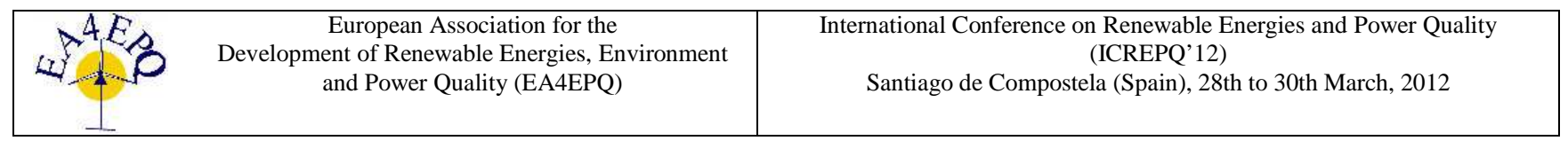

\title{
Development of 500[kWh] Lithium-Ion Battery Energy Storage System with 2[MVA] Power Conditioning System for Utility Interconnection
}

\author{
Kwang-Seob Kim ${ }^{1}$, Byung-Ki Kwon ${ }^{1}$ and Dong-Seok Hyun ${ }^{2}$ \\ ${ }^{1} \mathrm{R} \& \mathrm{D}$ Center of POSCO ICT \\ Techno-Complex, 126-16, Anam-Dong 5Ga, Sungbuk-Gu, Seoul, 136-701, Korea \\ Phone/Fax number:+82-2-3290-4457/+82-2-925-1812, e-mail: kkspn13k@ poscoict.com \\ ${ }^{2}$ Department of Electrical Engineering \\ HIT 401, Hanyang University, 17 Haengdang-dong, Seongdong-gu, Seoul, 133-791, Korea \\ Phone/Fax number:+82-2-2220-0341/+82-2-2290-0532, e-mail: dshyun@ hanyang.ac.kr
}

\begin{abstract}
Photovoltaic or wind turbine system installed around world has been increased dramatically in the last decade. Unfortunately, these systems are uncontrollable and dependent on environmental conditions i.e., sun or wind. As a consequence, increasing connections of those systems to the grid can lead to the stability problem of the utility network or even the failure because of their uncontrollability.

Battery energy storage system(BESS) can be used to control the output fluctuations of renewable sources. BESS consists of lithium-ion battery and power conditioning system(PCS). This paper gives critical aspects of design and control for a large capacity of battery system and PCS. Test results are also given as justification of design and control technology.
\end{abstract}

\section{Key words}

Battery energy storage system(BESS), Lithium-ion battery(LIB), Battery management system(BMS), Power conditioning system(PCS), Sinusoidal filter.

\section{Introduction}

Nowadays, climate changes have become worldwide issues as it threatens human beings. Fossil fuel such as oil, natural gas, and coal are main energy sources of power generation until now. Emission of greenhouse gases by fossil fuel is recognized as major cause of environmental problems. In addition, the increasing demand for energy can create more problems such as utility network instability and make the situation worse. So, renewable energy sources are seen as alternative to the traditional energy sources.

There are lots of renewable energy sources around world. Among those renewable sources, the main stream for renewable power generation are wind power, hydro power, and photovoltaic(PV) power. Especially, the wind power and PV power are increasing rapidly worldwide based on the low cost and government's programs, respectively. But these distributed renewable energy resources are characterized by uncontrollability. In other words, they have drawbacks that their output power is strongly dependent on environmental conditions i.e., wind and/or sun. The fluctuations of output power injected to the grid can lead to the instability of utility or even outages [1]. So, grid codes for connecting distributed resources tend to demand more stringent requirements. BESS can be used to solve the problems of output power variation of renewable sources.

This paper shows a large capacity BESS with 2[MVA] PCS to charge and/or discharge $500[\mathrm{kWh}]$ LIB system. Design problems and compliance with international standard for utility interconnection of BESS are discussed. Test results are also given to justify the design and control of a developed BESS.

\section{BESS}

BESS consists of 2[MVA] PCS and 500[kWh] LIB system as shown in Figure 1. LIB system can be connected in parallel so that a total capacity can be expanded up to 5[MWh]. Power management system(PMS) communicates with both PCS and all of LIB systems to control and to receive their status.

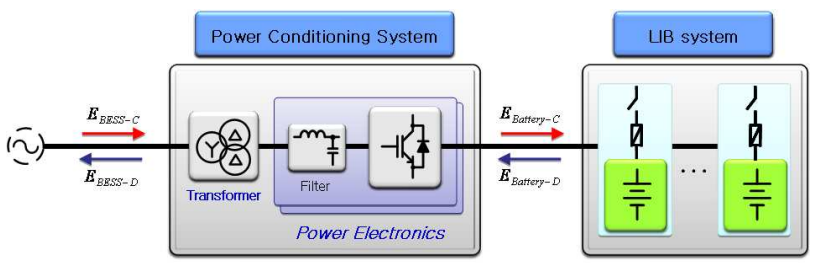

Fig. 1. Configuration of BESS.

\section{LIB system}

Photo of 500[kWh] LIB system is shown in Figure 2. It consists of 16 battery racks, BMS, heating ventilation airconditioning(HVAC) and protection system. It is containerized to be robust against environmental conditions such as dust, wind, rain, and snow etc. 


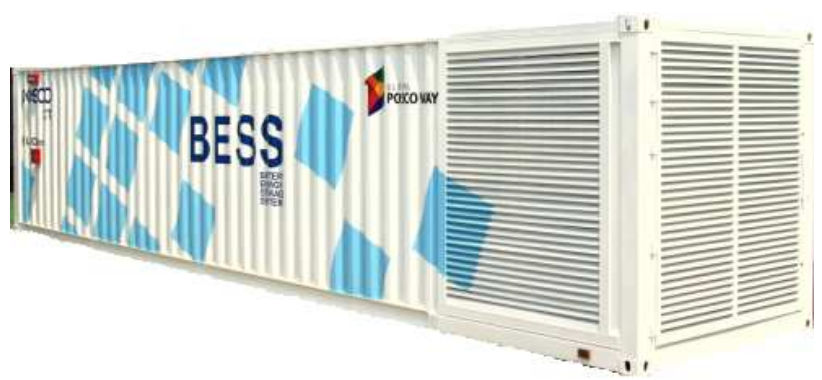

Fig. 2. Photo of 500[kWh] LIB system

\section{A. LIB cell}

LIB can be characterized by high energy density, no memory-effect, high output currents, and high efficiency compared to other battery technologies. Brief and general description on battery is given to help understanding and discussion of battery system. First, the capacity of battery is represented by C-rate. It is defined as the current level a battery can provide for one hour. Second, performances of cell can be described as follows. A charging and/or discharging C-rate is the maximum current a battery can supply without harmful effects or damage. State of charge(SOC) is defined as the percentage of how much capacity or energy battery cell release relative to the rated capacity. State of health $(\mathrm{SOH})$ is the percentage of a maximum capacity relative to its rated capacity. As battery cell charges and discharges repeatedly, the capacity of it is decreased to some extent. Depth of discharge(DOD) can be expressed as the percentage of discharged capacity relative to the rated capacity. Round trip efficiency is the percentage of discharged capacity relative to the charged capacity.

C-rate of lithium-ion battery cell used in this paper is $15 \mathrm{Ah}$. It can operate up to $4 \mathrm{C}$ equivalent to $60 \mathrm{~A}$. Typical characteristics of charging and/or discharging with respect to currents are shown in figure 3 .

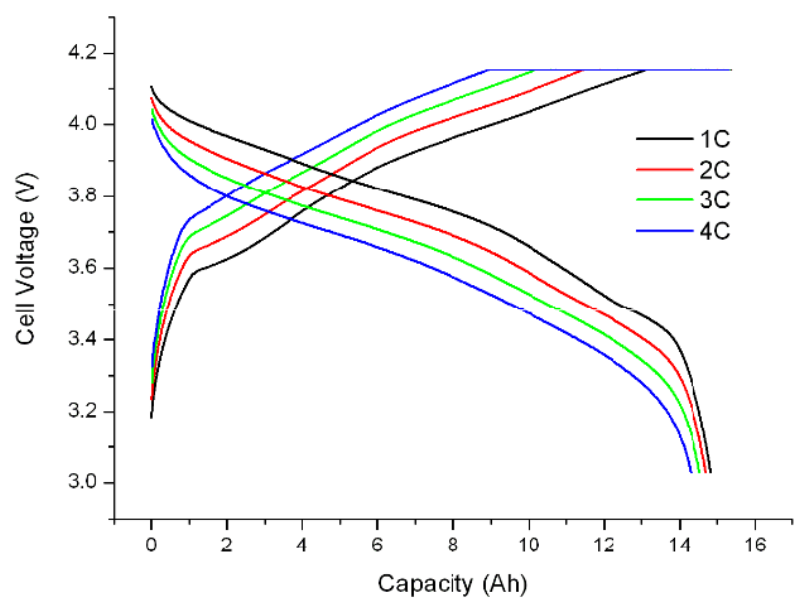

Fig. 3. charging/discharging characteristics of LIB

Battery is a nonlinear electrochemical device to store energy. It involves complex electrochemical process during charging and/or discharging. Electrochemical impedance spectroscopy(EIS) which is the Nyquist plot that shows the complex impedance of battery in a single curve over the entire frequency range is used to analyze the dynamic characteristic of battery. Typical plot is shown in figure 4 . Its characteristics can be divided into three regions with respect to frequency range. Low frequency region ranging from $\mathrm{mHz}$ to a few $\mathrm{Hz}$ shows linear ohmic characteristics. Half circle in the middle range of $\mathrm{Hz}$ to $\mathrm{kHz}$ is similar to $\mathrm{RC}$ parallel circuit and represents the charge transfer and electrochemical double layer effect. High frequency range from $\mathrm{kHz}$ to $\mathrm{MHz}$ shows the conductance and skin effect. Equivalent electrical network of battery based on this plot can be made as shown in figure 5 .

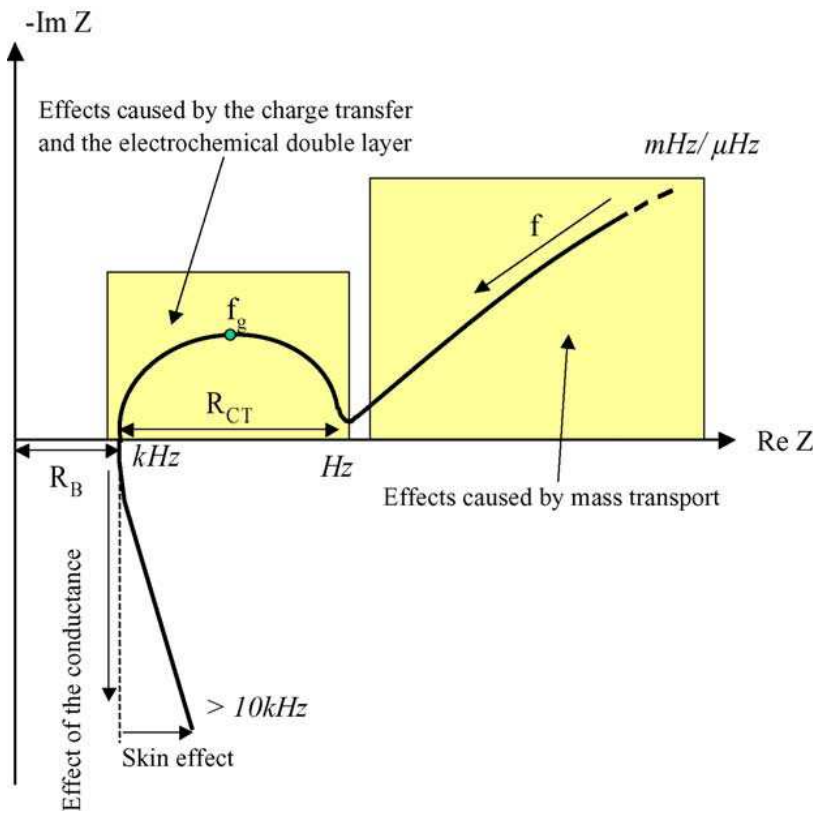

Fig. 4. Typical Nyquist plot of a battery cell

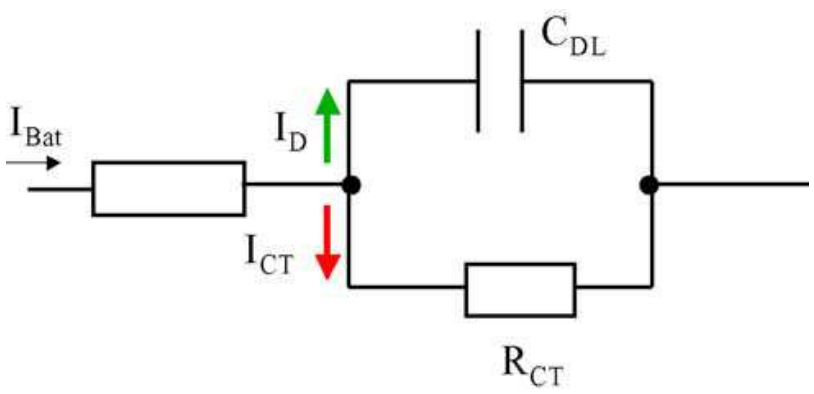

Fig. 5. equivalent circuit of a battery cell

\section{B. Battery rack}

One rack has 24 modules connected in series. Each module is constructed with 8 cell groups which have 3 LIB cells connected in parallel. The nominal voltage of each cell is $3.75 \mathrm{~V}$ and its operating range is from $3.0 \mathrm{~V}$ to $4.15 \mathrm{~V}$. So, nominal output voltage is $720 \mathrm{~V}$ because one rack has 192 cell groups connected in series.

As a rack is charged, the smallest capacity of cell reaches its maximum voltage even though other cells have not reached its maximum voltage. When it is discharged, the smallest capacity of cell touches its minimum voltage with other cells having enough marginal voltage. As mentioned above, each cell has to be operated within safe voltage range to protect cell itself. So, the capacity of a rack is not equal to the sum of all the cells. The effective capacity of a rack is reduced to the extent that the smallest capacity cell of a rack is protected from over/under-voltage. Battery cell screen which selects 
cells having similar capacity and internal impedance is very important to maximize utilization of battery cells.

Depth of discharge(DoD) which is the operating voltage range of LIB system should be selected carefully to consider trade-offs between life expectancy of battery and utilization of battery capacity. As DoD gets deeper, the amount of electricity which can be stored in a battery is increased but the life span of battery is decreased rapidly.

Terminal voltage of battery system varies when it charges or discharges as shown in test results. These variations of terminal voltage are due to internal impedance and SOC [2]. It also means terminal voltage variation can not be negligible. Variations of terminal voltage have to be taken into consideration as design parameters of PCS because dc-link voltage of inverter shown as power electronics in figure 1 is the same to terminal voltage of battery rack. Nominal voltage of inverter or transformer for utility interconnection should be reduced as discharging rate of battery rack increases.

\section{BMS}

Although LIB has lots of advantages, it has severe drawback of safety. It can be destroyed and/or even exploded if the charging voltage goes beyond the critical level. That's why BMS is needed to monitor and protect LIB cell. It measures temperature, voltage, and current of LIB cell. Then, it conducts analysis and/or diagnosis to calculate state of charge(SOC) and/or state of health(SOH) based on measured data. If battery cell is out of nominal condition, it takes measure for protection.

BMS for $500[\mathrm{kWh}$ BESS has a hierarchical structure which is composed of three kind of BMS, i.e. unit BMS, rack BMS, and system BMS. Unit BMS measures temperature and voltage of each cell and communicates with a rack BMS to send measured data. A rack BMS measures current of each rack and receive measured data from unit BMS. It calculates SOC and makes decisions of over/under-voltage and over-temperature to protect battery cell. It also communicates with system BMS to exchange data and control command. System BMS acts as interface in order to give information from all the rack BMS to a high level control system. It communicates with power management system(PMS).

\section{HVAC \& Protection}

The performance of LIB is optimized under recommended operating conditions. So, HVAC operates to control the temperature and humidity of LIB system. In case of LIB explosion, inert gases are ejected to extinguish fire.

\section{PCS for Utility Interconnection}

PCS consists of water cooled PWM inverter, multiwinding step-up transformer, medium voltage switchgear, and cooling equipment. All the equipments are included in a container in order to make transportation and installation easy. Outlook of 2[MVA] PCS is shown in figure 6.

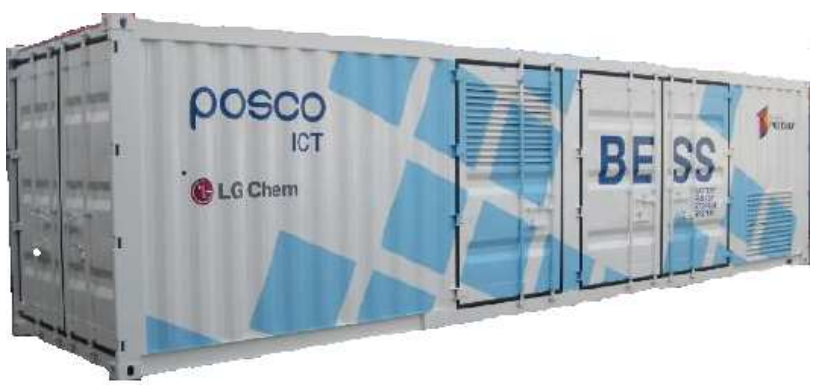

Fig. 6. Photo of 2[MVA] PCS connected to 22.9[kV] distribution line.

\section{A. Battery Interface}

PCS plays a role of charging/discharging a large capacity battery according to the command of PMS. It should protect battery from being overcharged and being discharged below critical voltage level.

It has three operation modes to charge and/or discharge battery. Constant current(CC) mode can be operated from minimum terminal voltage which is $576 \mathrm{~V}$ to maximum terminal voltage which is $797 \mathrm{~V}$. It draws constant current from battery. Constant voltage(CV) mode is activated as terminal voltage reaches maximum voltage and its charging current decay slowly according to SOC. In a constant power( $\mathrm{CP})$ mode, not the current or voltage but power of battery is controlled constant. So, the input or output current of battery varies according to terminal voltage. In other words, input current decreases as battery voltage goes up by charging and output current increases as its voltage comes down by discharging. $\mathrm{CP}$ mode is usually adopted in an application where control of rampup or ramp-down rate of generator is important. WT and $\mathrm{PV}$ is one of such applications.

\section{B. Utility Interface}

Recently, grid code tends to be stringent as lots of distributed sources are increasing. PCS should comply with utility interconnection standard such as IEEE std. 1547. It has to perform fast and robust control to meet those requirements of grid code.

It adopts a sinusoidal output filter to reduce harmonic currents injected into utility. LC circuit is used as an output filter and active control algorithm of damping LC resonance is also needed. Several design guides and control schemes for LCL filter are suggested [3]-[5].

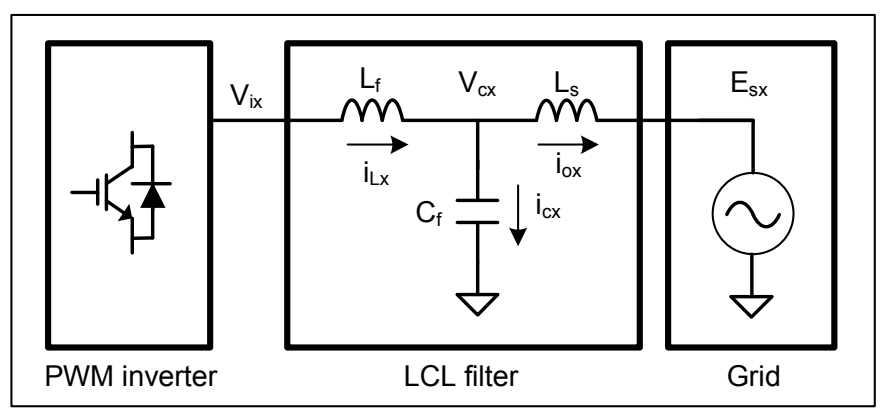

Fig. 7. Circuit diagram of LCL filter

Equation (1) can be written for each phase of LCL filter in Fig. 7, where $\mathrm{x}$ represents one of the three phases $\mathrm{u}, \mathrm{v}$, or $\mathrm{w}$. 
$v_{i x}=L_{f} \cdot i_{L x}^{\prime}+v_{c x}$

$v_{c x}=L_{s} \cdot i_{o x}^{\prime}+E_{s x}$

$i_{c x}=C_{f} \cdot v_{c x}^{\prime}$

$i_{L x}=i_{c x}+i_{o x}, \quad(x=u, v, w)$

LC filter can be transformed as equation (2) in a stationary reference frame.

$v_{i D Q}=\frac{v_{c D Q}^{\prime \prime}}{\omega_{n}^{2}}+v_{c D Q}+L_{f} \cdot i_{o D Q}^{\prime}$

$\omega_{n}$ in (2) is the natural frequency of LC filter and is represented as (3).

$$
\omega_{n}=\frac{1}{\sqrt{L_{f} \cdot C_{f}}}
$$

Equation (2) is transformed as (4) in a synchronous reference frame where $\omega$ is angular frequency of synchronous reference frame.

$$
\begin{gathered}
v_{i d q}=\frac{v_{c d q}^{\prime \prime}}{\omega_{n}^{2}}+v_{c d q}-\frac{\omega^{2}}{\omega_{n}^{2}} \cdot v_{c d q}+j \frac{2 \omega}{\omega_{n}^{2}} \cdot v_{c d q}^{\prime} \\
\quad+L_{f} \cdot i_{o d q}^{\prime}+j \omega L_{f} \cdot i_{o d q}
\end{gathered}
$$

Equation (4) is rearranged and simplified to make the typical second order low pass filter(LPF). All of them except the first two terms in the right of (4) are moved to left and damping term is added to both sides of (4). Equation (5) is the expression, where $\zeta$ is a damping ratio of second order LPF. Load currents are omitted from (5) because it is compensated as feed-forward term.

$$
\begin{aligned}
& v_{i d q}+\frac{2 \zeta}{\omega_{n}} v_{c d q}^{\prime}+\frac{\omega^{2}}{\omega_{n}^{2}} \cdot v_{c d q}-j \frac{2 \omega}{\omega_{n}^{2}} \cdot v_{c d q}^{\prime} \\
& =\frac{v_{c d q}^{\prime \prime}}{\omega_{n}^{2}}+\frac{2 \zeta}{\omega_{n}} v_{c d q}^{\prime}+v_{c d q}
\end{aligned}
$$

The derivative of capacitor voltage is approximated as (6), where $T_{s}$ is a sampling time, $\alpha$ is the unit-step response for one sampling time, and $v_{c d q}^{*}$ is the reference of output filter capacitor.

$v_{c d q}^{\prime} \approx \frac{\alpha}{T_{s}} \cdot\left(v_{c d q}^{*}-v_{c d q}\right)=K \cdot\left(v_{c d q}^{*}-v_{c d q}\right)$

Equation (7) is the reference of inverter output voltage which is calculated from (5), (6).

$$
\begin{gathered}
v_{i d q}=v_{c d q}^{*}-\frac{2 \zeta}{\omega_{n}} K\left(v_{c d q}^{*}-v_{c d q}\right)-\frac{\omega^{2}}{\omega_{n}^{2}} \cdot v_{c d q} \\
+j \frac{2 \omega}{\omega_{n}^{2}} K\left(v_{c d q}^{*}-v_{c d q}\right)
\end{gathered}
$$

Current control is implemented easily in a synchronous frame because the capacitor voltage of output filter can be controlled as a second order LPF by using (7). Figure 8 shows overall structure of current controller. IP-type current controller is adopted and FC is a filter controller of equation (7). It also includes decoupling voltage terms and feed-forward voltage.

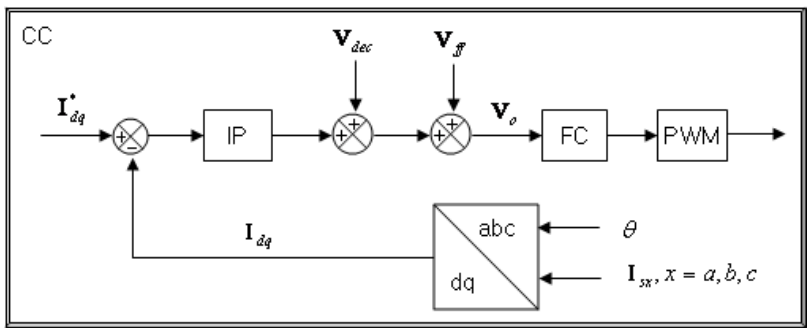

Fig. 8. Structure of current controller

One of most important issues of PCS is how to find information of grid, i.e. phase angle, frequency, and voltage magnitude, because calculation and control of active/reactive power, transformation of variables are based on the grid information. Many technologies have been presented to deal with grid voltage distortion, and unbalanced voltage as well [6],[7]. Among them, phase locked loop(PLL) in a synchronous reference frame is adopted as shown in figure 9. As shown, grid voltage is aligned to q-axis in a synchronous reference frame

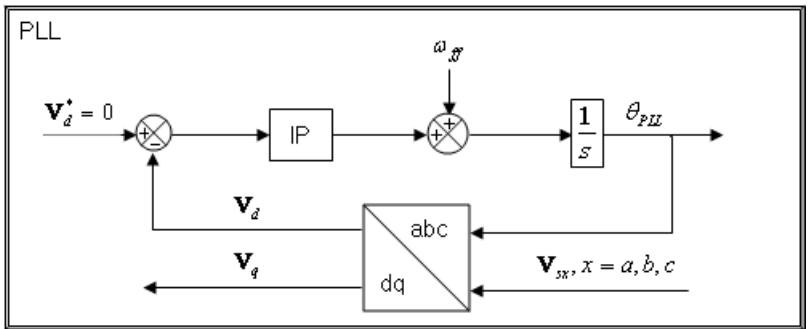

Fig. 9. PLL to detect utility information

\section{Test Results}

Parameters of PCS output filter are shown in Table I. Table I.

\begin{tabular}{|l|c|l|}
\hline Switching freq. & $4[\mathrm{kHz}]$ & Bold centred \\
\hline Lf & $20[\mathrm{uH}]$ & Centred \\
\hline Cf & $700[\mathrm{uF}]$ & Delta connection \\
\hline Ls & $20[\mathrm{uH}]$ & \\
\hline$\% \mathrm{Z}$ & $6[\%]$ & $2[\mathrm{MVA}] \mathrm{Tr}$. \\
\hline
\end{tabular}

Figure 10 shows terminal voltage of LIB system varies as it charges or discharges. As terminal voltage approach to maximum value, charging mode of PCS change from CC to $\mathrm{CV}$ and charging currents decay slowly.

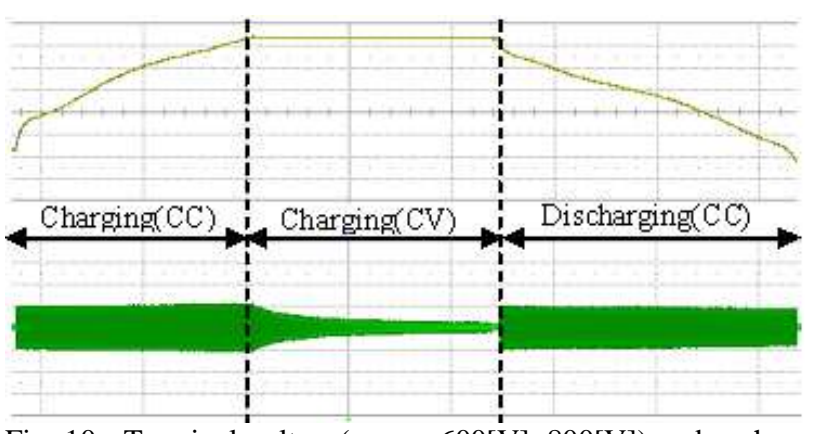

Fig. 10. Terminal voltage(upper, 600[V] 800[V]) and u-phase current of $22.9[\mathrm{kV}]$ (bottom, 20[A/div]) waveform, [1000s/div]. 
Figure 11 shows transient output voltage waveforms of PCS in the beginning stage of synchronization. Output voltage builds up to grid voltage under LC filter control and the phase difference is getting small so that there is little phase difference. Synchronization is completed when the difference of voltage magnitude, phase, and frequency stay specific values defined in IEEE std. 1547 for specified duration. As a circuit brake to connect with utility network is closed after synchronization, it draws little inrush currents from grid.

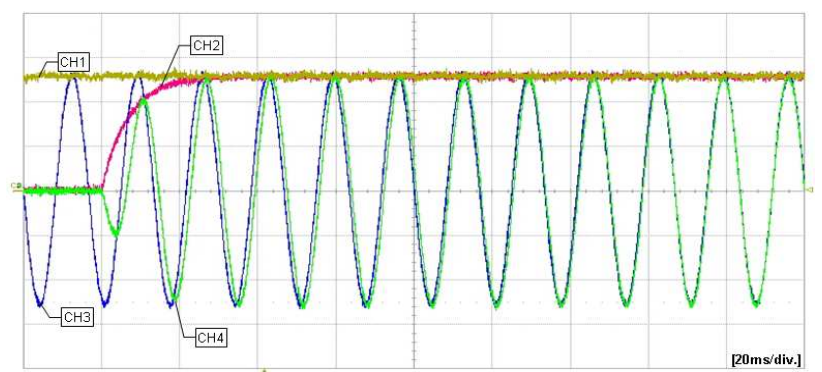

Fig. 11. Synchronization waveforms of PCS, CH1(q-axis grid voltage), $\mathrm{CH} 2$ (output voltage), $\mathrm{CH} 3$ (u-phase grid voltage), CH4(u-phase output voltage), [0.4pu/div], [20 msec/div].

Utility voltage and Output current waveforms are close to sinusoidal waveform and power factor is controlled exactly unity as shown in figure 12 . It shows that output filter control and current control work well at rated load. THD of output currents are measured by power analyzer of PZ4000. Result shows that maximum THD is below 3\% at rated condition.

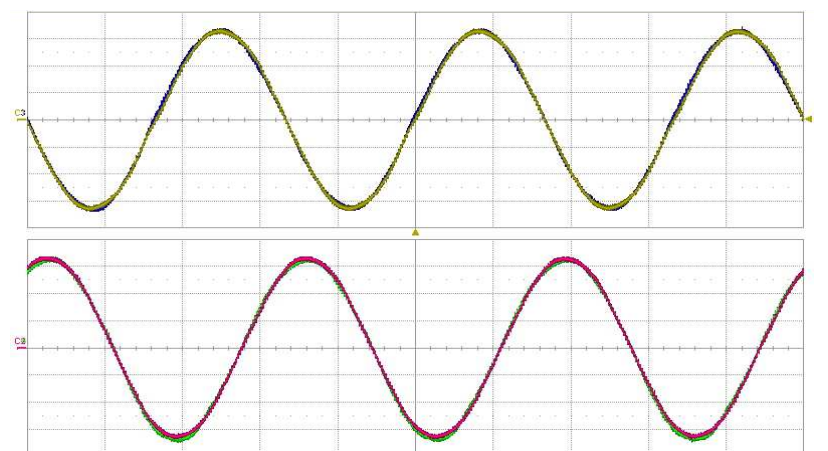

Fig. 12. U-phase voltage and current(upper) and v-phase voltage and current (bottom) waveforms, [0.3pu/div], [5ms/div].

\section{Conclusion}

Design and control strategy for a large capacity BESS is presented. Structure of $500[\mathrm{kWh}]$ battery system and its operational characteristics are also discussed. PCS for battery charging and/or discharging has three operation mode to deal with battery restriction and protection. It also should comply with international standard for utility interconnection and adopts LCL output filter to meet harmonic requirements. Control algorithms are implemented on a DSP board and various tests are carried out. Test results are presented to verify the effectiveness of design and control of BESS.

\section{References}

[1] F. Blaabjerg and R. Teodorescu, M. Liserre, Adrian V. Timbus, "Overview of Control and Grid Synchronization for Distributed Power Generation Systems", IEEE Trans. On Industrial Electronics.vol.53, no.5.2006., pp. 1398-1409.

[2] Stephan Buller, Member, IEEE, Marc Thele, Rik W. A. A. De Doncker, Fellow, IEEE, and Eckhard Karden, Member, IEEE, "Impedance-Based Simulation Models of Supercapacitors and Li-Ion Batteries for Power Electronic Applications", in IEEE TRANSACTIONS ON INDUSTRY APPLICATIONS. VOL. 41, NO. 3, MAY/JUNE 2005, Vol. 1, pp. 742-747.

[3] Juergen K. Steinke, "Use of an LC Filter to Achieve a Motor-friendly Performance of the PWM Voltage Source Inverter", IEEE Transactions on Energy Conversion, Vol. 14, No. 3, September 1999, pp. $649 \sim 655$.

[4] Marco Liserre, Member, IEEE, Frede Blaabjerg, Fellow, IEEE and Steffan Hansen, Member, IEEE, "Design and Control of an LCL-Filter-Based Three-Phase Active Rectifier", IEEE Trans. On Industry Applications, VOL. 41, NO.5, SEPTEMBER/OCTOBER 2005, pp. 1281-1291.

[5] Kamran Jalili, Steffen Bernet, Member, IEEE, "Design of LCL Filters of Active-Front-End Two-Level Voltage-Source Converters", IEEE Trans. On Industrial Electronics, VOL. 56, NO. 5, 2009, pp. 1674-1689.

[6] Sang-Joon Lee, Jun-Koo Kang, Seung-Ki Sul, "A new phase detecting method for power conversion systems considering distorted conditions in power system", Industry Applications Conference, vol. 4, 1999, pp.2167 - 2172.

[7] Se-Kyo Chung, Member, IEEE, “A Phase Tracking System for Three Phase Utility Interface Inverters", IEEE Trans. On POWER ELECTRONICS, VOL. 15, NO. 3, MAY, 2000, pp. 431-438. 